\title{
A Machine LeARning Ontology
}

\author{
Juliao Braga \\ Faculdade de Computação e Informática \\ Universidade Presbiteriana Mackenzie \\ Sao Paulo, SP, Brazil \\ juliao@braga.eti.br
}

\author{
Joaquim L R Dias \\ Instituto Superior Técnico \\ Universidade de Lisboa \\ Lisbon, PT \\ joaquim.dias@tecnico.ulisboa.pt
}

\author{
Francisco Regateiro \\ Instituto Superior Técnico \\ Universidade de Lisboa \\ Lisbon, PT \\ francisco.regateiro@ist.utl.pt
}

July 16, 2021

\begin{abstract}
This paper describes the creation of an ontology to represent the knowledge around the Machine Learning discipline. Protégé 5 was used, which produces results suitable for agents developed by software and for humans. The knowledge created by Protégé is explicit and Protégé has itself inference machines capable of producing implicit knowledge. The resources available in Protégé 5 are displayed and the ontology is made available for public use, in all of its versions.
\end{abstract}

\section{Introduction}

Ontology was defined in 1993 by Gruber and in 1997 this definition was adjusted more appropriately by Borst [1] apud $[2,3]$. In 1998, Studer and others adjusted the two definitions in the following definition proposal: "An ontology is a formal, explicit specification of a shared conceptualization" [4] [1]. Several authors have refined the definitions over time to indicate, more clearly, that an ontology is a formally-defined vocabulary for a particular domain of interest used to capture knowledge about that (restricted) domain of interest. So, an ontology describes the concepts in the domain and also the relationships that hold between those concepts [5]. The inclusion of a domain in the definition made it easier to understand the meaning of ontology. In this way and finally, it was defined ontologies as describing concepts and relations in a specific domain are necessary for Knowledge Representation (KR) and Knowledge Exchange (KE) [6].

The present work considers a specific ontology for a repository of explicit knowledge on a well-defined machine learning domain represented by specifications that allow to extend this repository with implicit knowledge obtained through the action of a reasoning engine [7]. This knowledge base is called Machine Learning Ontology (MLOnto) and can be used by humans and software-based agents (agents). The agents, in the context of this work, are part of the model named Autonomous Architecture Over Restricted Domains (A2RD) that depend on a knowledge base, one of the components that are hosted in a support environment named Structure for Knowledge Acquisition, Use, Learning and Collaboration (SKAU) whose ecosystem is visible in Figure 1 [8].

MLOnto is built using Protégé 5 (Protégé), an ontology editor, which uses the Web Ontology Language (OWL) which, in turn, adopts Manchester syntax ${ }^{1}$ [11] [12]. Among the various languages available to represent knowledge, OWL brings together a set of favorable facilities because it has a high degree of expressiveness (Figure 2) through a reasonably compact syntax, precise semantics under its formalism and easy to be analyzed by reasoning mechanisms [13] [14]. Protégé was created at Stanford University and is very popular in the field of Semantic Web. Protégé is free,

\footnotetext{
${ }^{1}$ https://www.w3.org/TR/ow12-manchester-syntax/
} 


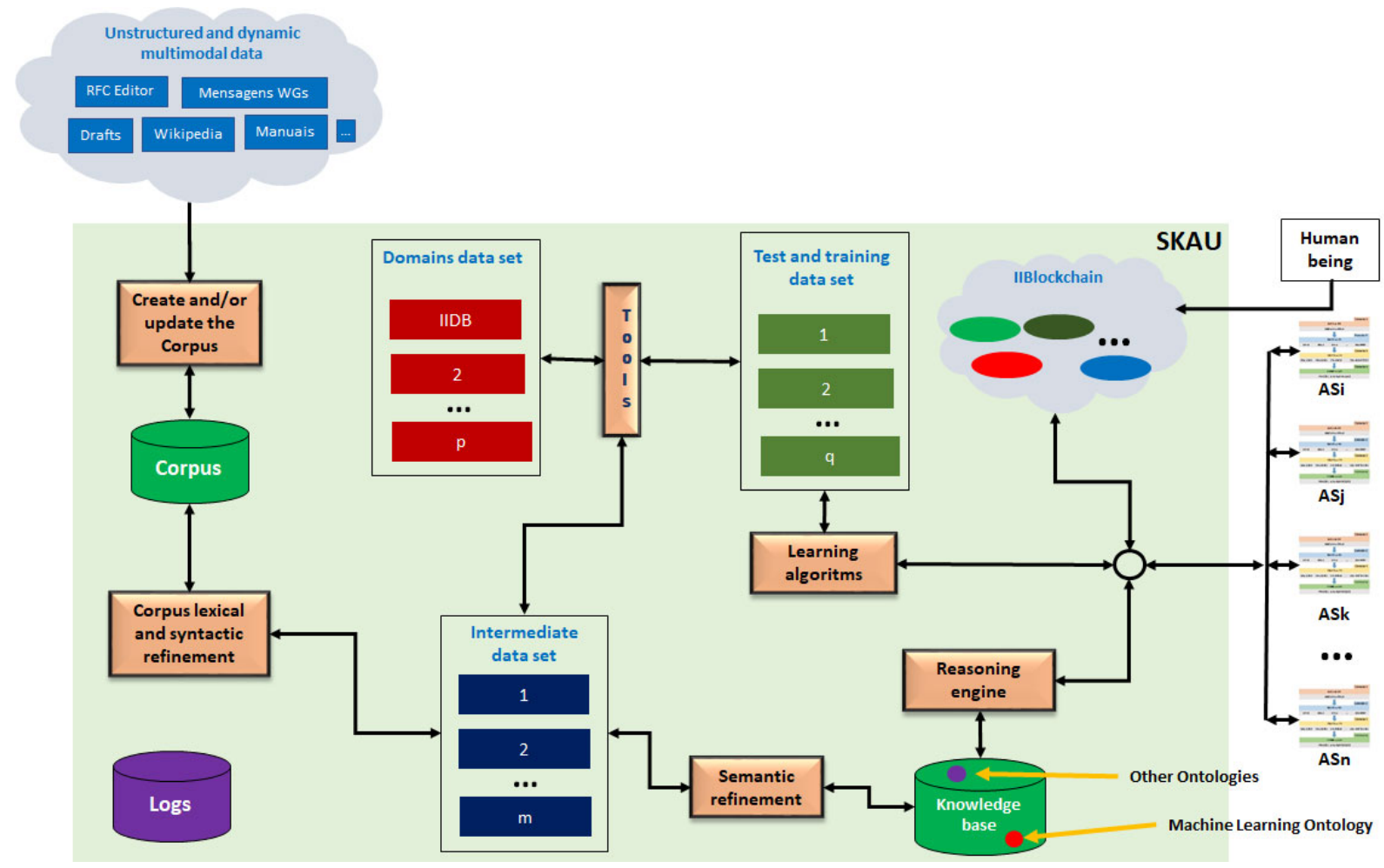

Figure 1: This image shows the SKAU environment, support for agents of the A2RD models, where the Knowledge Base displays the position of Machine Learning Ontology and other ontologies, also. The other items that appear in the image of this figure are components that complete the SKAU environment that, although important are outside the scope of this paper. Adapted from [9] or [10].

developed in Java and its source code is released under a free license (the Mozilla Public License). Protégé can read and save ontologies in most ontologies formats: RDF, RDFS, OWL, etc. It is recognized for its ability to work on large Ontologies [11] [6] [15].

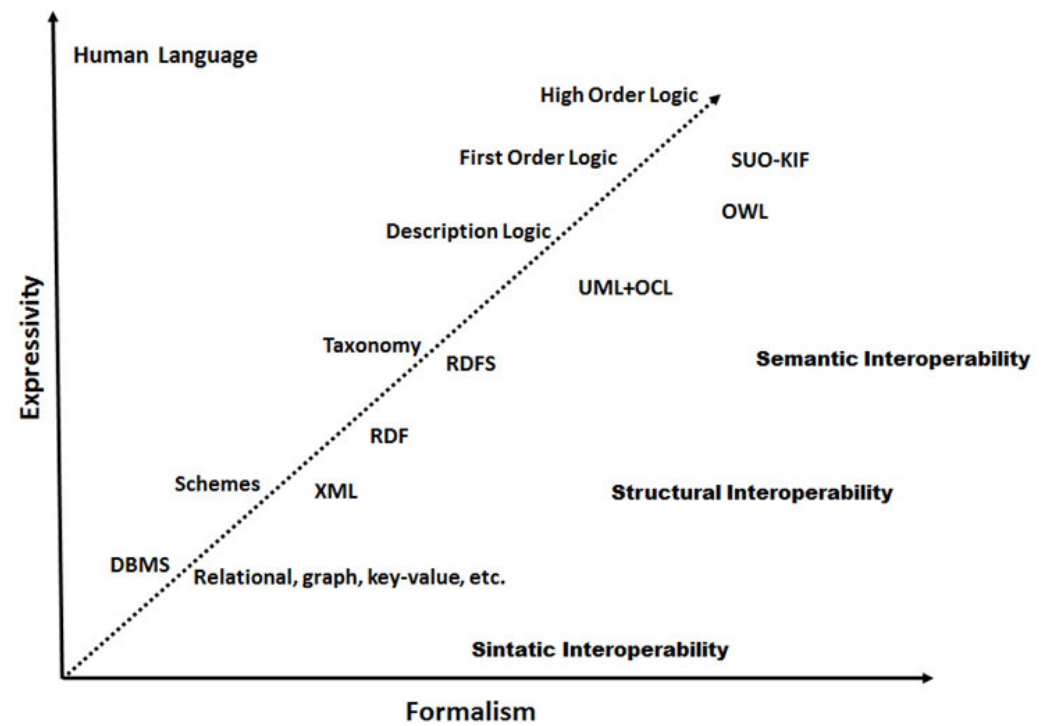

Figure 2: Languages Expressiveness. Source: [16] 
This work is divided into six sections, including this Introduction. Section 2 describes the related work. Section 3 describes the MLOnto architecture by identifying each of its seven highest-level classes. Section 4 describes its current implementation structure at Protégé. The section 5 establishes the conclusions and future work and the last section 6 informs about the availability of the latest version of the ontology database.

\section{Related Works}

The use of Machine Learning to build Ontologies or a subset of Ontologies has been common in the literature [17, 18]. Automatic processes to produce ontologies are a constant presence, such as the work of capturing acronyms from Request for Comments (RFCs) corpus, documents produced by the Internet Engineering Task Force (IETF) [9]. There are many works related to the Ontologies construction that include methodologies using Protégé, in the most diverse domains and applications [e.g. [19, 20, 21, 22, 23]]. However, initiatives for the construction of Ontologies that express the knowledge of the domain of Machine Learning have few references on very specific sub-domains $[24,25,26]$.

Lately, new area is being proposed: ontology learning using techniques of Natural Language Processing, AI and machine learning [27, 28, 29]

\section{The MLOnto Architecture}

Recently, Vaishak Belle and Ioannis Papantonis [30], divided multi-stakeholders in understanding machine learning models into five groups, when they observed that ML models are increasingly deployed in a wide range of companies: (a) data scientists, (b) business owners, (c) model risk analysts, (d) regulators and (e) consumers, characterizing them in a didactically clear way.

Concerns and experiences like those of these authors, stimulated the creation of the first stage of MLOnto, adding the fact that another interested party, (f) the agents (as shown in Figure 1), must follow the changes and the complexity of the knowledge domain that populates the machine learning discipline..

The first stage of the construction of the MLOnto is manual and we considered the seven main classes presented in Figure 3, whose details are discussed in the subsection below.

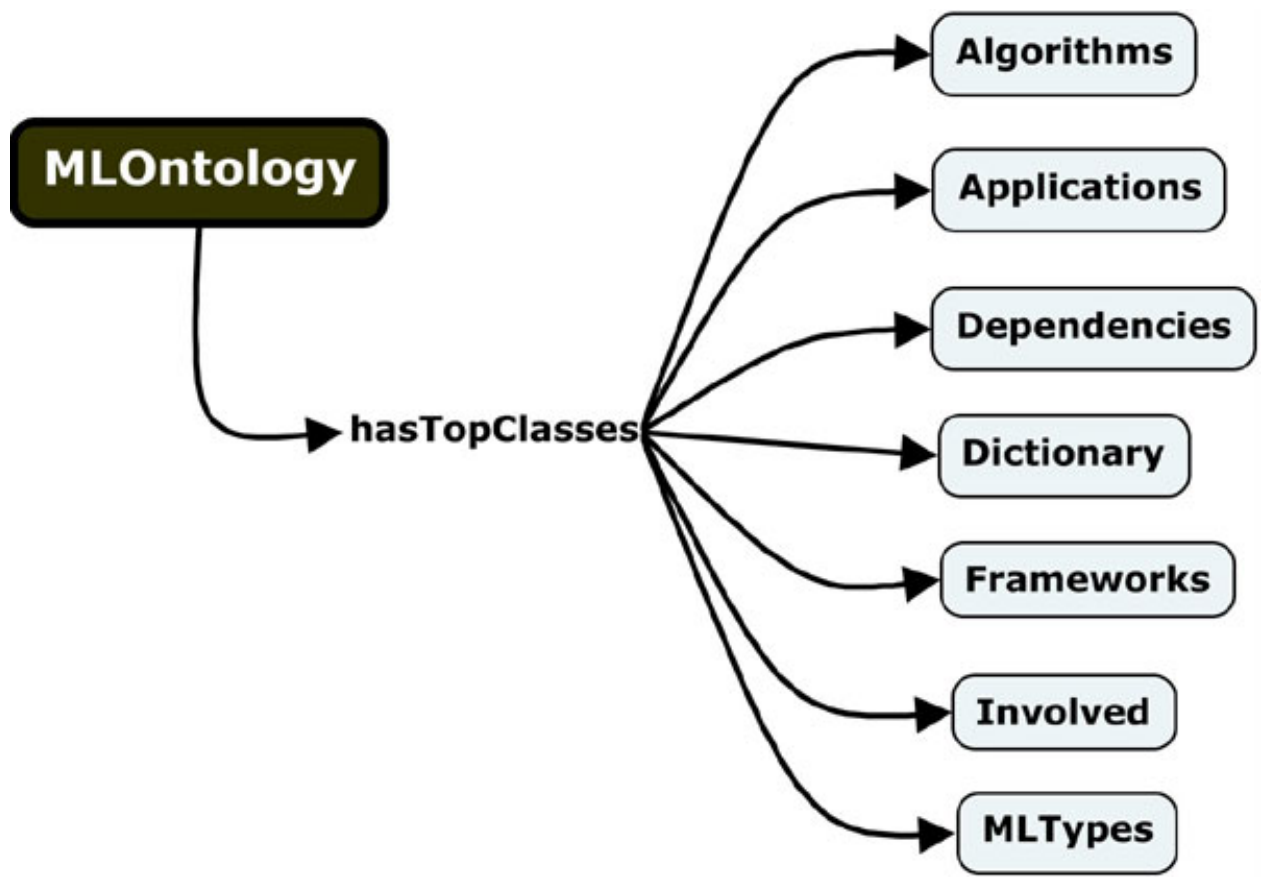

Figure 3: The MLO highest level classes

These seven classes were considered, for now, sufficient to describe the domain of knowledge that encompasses the discipline of Machine Learning considered a branch of Artificial Intelligence (AI). 


\subsection{The MLOnto Highest Level Classes}

The main characteristics of these seven highest level classes are:

- Algorithms: A set of rules that precisely defines a sequence of operations, which would include all computer programs, including programs that do not perform numeric calculations.

- Applications: the action of putting something into operation.

- Dependencies: What are the prerequisites for solving an algorithm or problem and what are the requirements for a tool to be executed.

- Dictionary: Acronyms, nicknames and phrases for which an interpretation is required.

- Frameworks: A set of tools and associated utilities for solving and implementing algorithms, problems or applications

- Involved: People or institutions that created and improved the available resources to facilitate the use and application of Machine Learning.

- MLTypes: Usual classification of Machine Learning algorithms. Although, more recently, the literature is adopting only three types of Machine Learning - (a) Supervised Learning, (b) Unsupervised Learning, (c) Reinforcement Learning - we are initially adopting two additional types, (d) AutoML, (e) Semi-Supervised Learning (Figure 4).

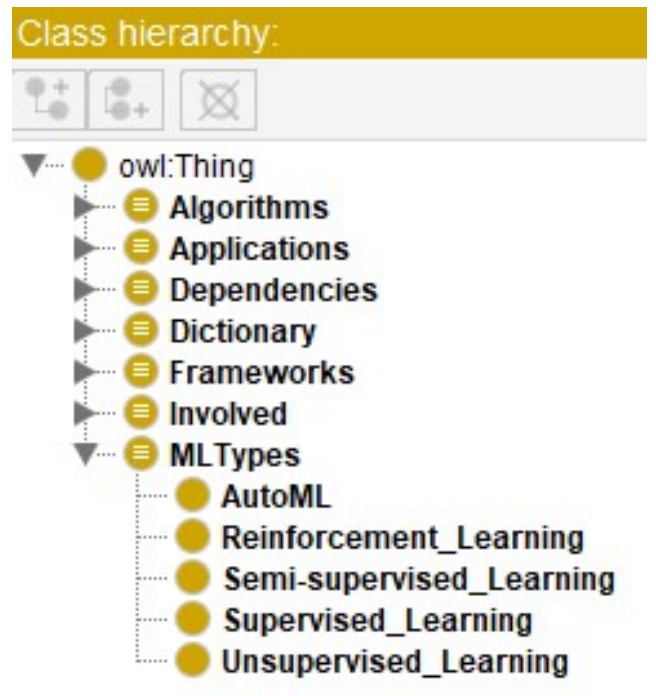

Figure 4: Machine Learning Types in MLOnto

\section{The implementation structure}

The resources available in Protégé will be used extensively, such as the Annotations (Figure 5) that allow indicating the references and clarify or justify non-traditional choices.

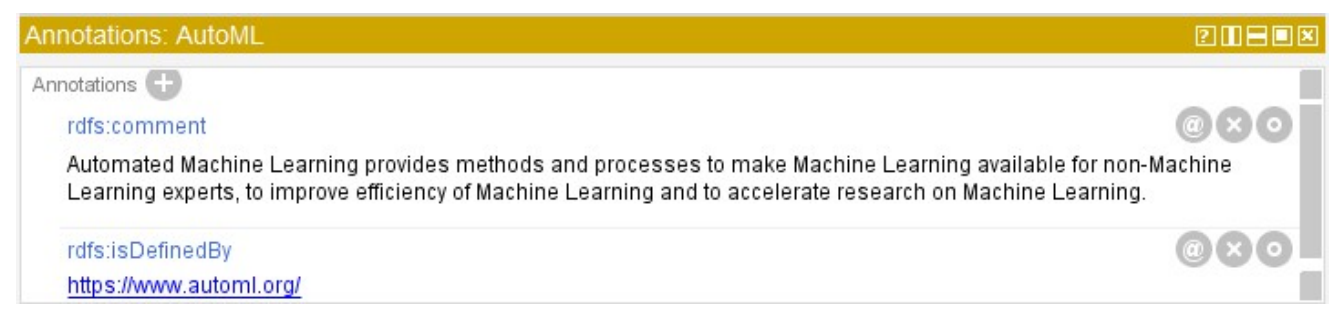

Figure 5: Annotation implementation in Protégé 
The contents added in Annotations are axioms and therefore react naturally under the mechanisms of inference or search performed by humans or agents. Additionally, properties that can be included in Annotations are dynamic and can be added at the discretion of the ontology administrator. The Figure 6 shows six properties added specifically for MLOnto's interests.

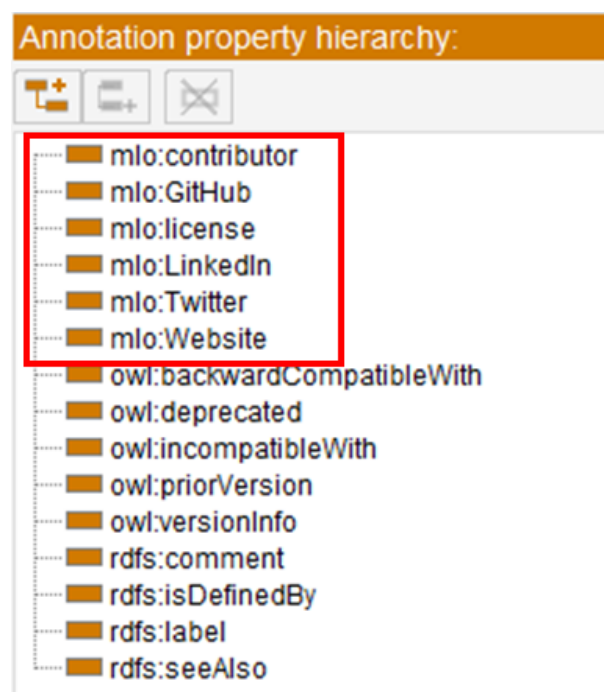

Figure 6: MLO especific Annotations properties

Protégé produces an OWL file that serves agents made by software, also. OWL serves human beings, too, but the Protégé interface is better prepared to facilitate human understanding of ontology. For example, in Figure 7, a view centered on the Algorithms class displays the branches graphically.

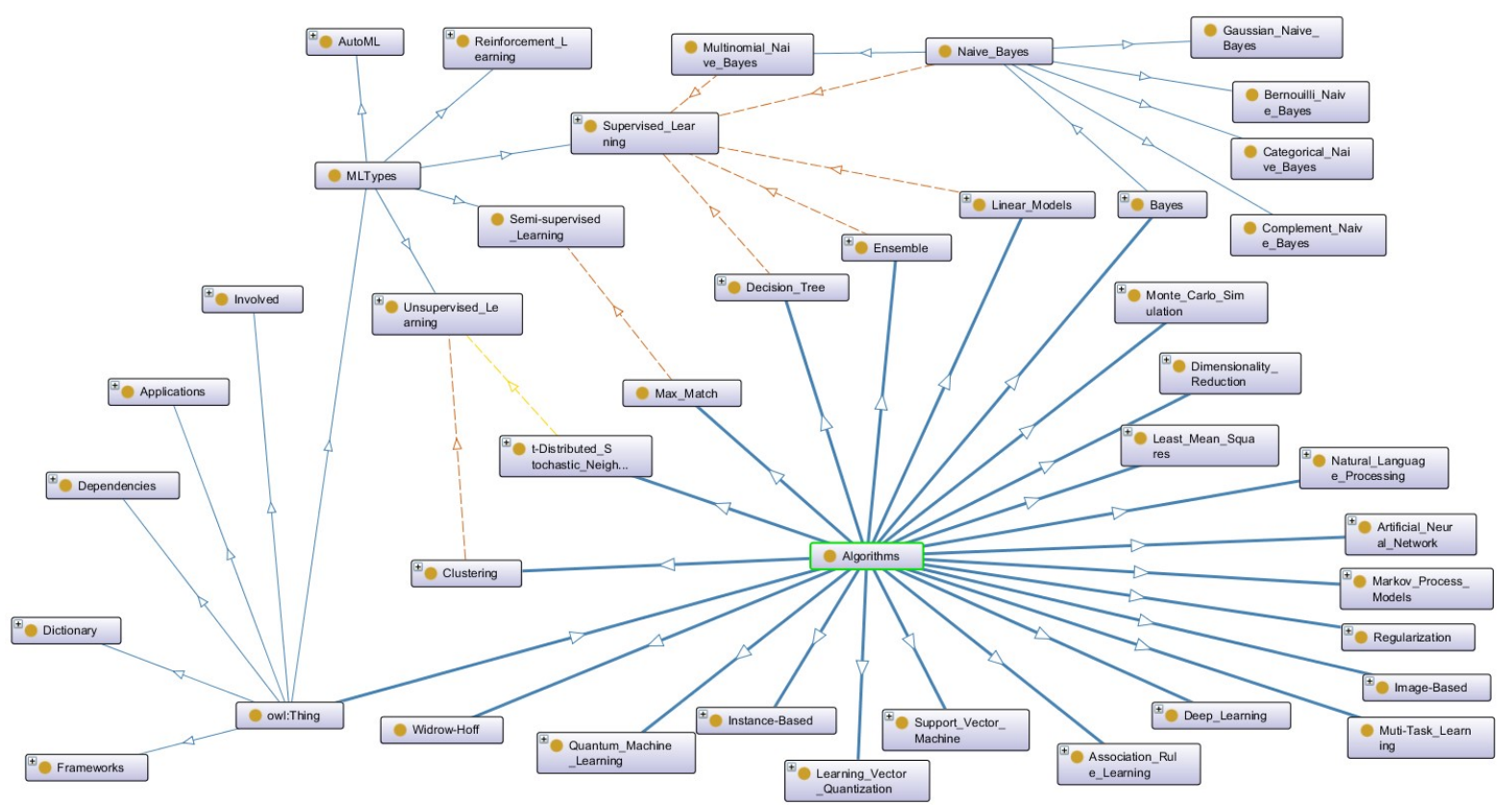

Figure 7: Partial graphic from the MLOnto with emphasis on the Algorithms class

In addition, Protégé's features include facilities that allow MLOnto, to debugging allowing corrections and refinements to be made easily with the created ontology. 


\section{Future Works}

The ontology is dynamic, as the knowledge of the Machine Learning domain is constantly renewed. A mechanism is needed to ensure the systematic updating of MLOnto in view of the Never-Ending Learner paradigm [31]. Specialized languages in learning that never-ends, have been proposed for applications in large domains, have been studied but can be thought of applying over restricted and very narrow domains, such as in the SciPy tool facility updates [32] Also, we must consider that the application referred to as Lifelong Machine Learning (LML), considers systems that can learn many tasks from one or more domains over its lifetime [33]

It would be appropriate for issues related to ethics in the construction of algorithms and the implementations that associate transparency, law and individual or group values of human society to be incorporated and characterized in MLOnto [34].

It is necessary to advance research and efforts in automatic processes for building ontologies from unstructured bases, mainly, encouraging the continuity of similar projects, which already exist [35]. Additionally, along the same lines and concerns, innovative research must be strongly considered [36].

There are many inference techniques available and it is necessary to research which ones are most appropriate to the various ontologies and identify the respective applications. Protégé has several inference mechanisms that make testing easy to identify the most appropriate ones.

Ontology Evaluation is the problem of assessing a given ontology from the point of view of a particular criterion of application, tipically in order to determine which of several ontologies would best suit a particular purpose [37].

In the sequence, it is admitted the proposal to use the Ontop ${ }^{2}$, a popular open-source Virtual Knowledge Graph (VKG) system that can expose heterogeneous data sources as a unified knowledge graph [38] [39]. Ontop has a Protégé plug-in facility that will be very useful for SPARQL applications. Also, agents of the A2RD who will benefit from the SKAU environment will have access to SPARQL Ontop facilitating search and reasoning mechanisms (Figure 1).

\section{Conclusions}

An ontology like this, in the domain of Machine Learning is a practically endless task. From a certain point on. the authors' concerns are emphatic in the questions of quality and reliability of the data that will be included. As an example it is worth mentioning the choice to include the techniques and algorithms used in applications under the domain of Internet Infrastructure. Our team considered that this task should be preceded by a literature review (not necessarily a systematic literature review) with which would be obtained the primary works that could confirm the additions to the current ontology.

The example given above, associated with the Internet Infrastructure, was made the choice to include the machine learning applications over the Autonomous Systems sub-domain, name given to the computer networks that represent, together, the Internet [10]. Thus, it was a matter of doing a literature review (non-systematic) to survey the primary studies that could produce the data and qualify them.

This paper has been updated by $[40]^{3}$.

\section{Data availability}

The latest version of the MLOnto database generated by the current study is publicly available on Open Science Foundation $^{4}$ (OSF) [41]. Some metrics from MLOnto in Protégé is available in Figure 8. Content license: Creative Commons 4.0 BY.

\footnotetext{
${ }^{2}$ https://ontop-vkg.org/guide/

${ }^{3}$ https://sol.sbc.org.br/index.php/wpietf/article/view/15778/15619

${ }^{4}$ https://osf.io/chu5q/
} 


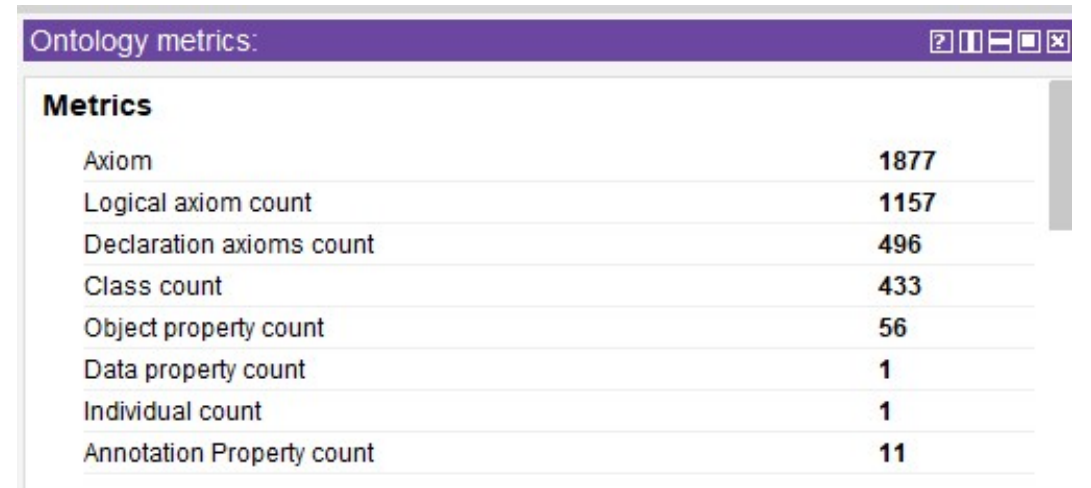

Figure 8: Some metrics from MLOnto in Protégé

\section{References}

[1] Nicola Guarino, Daniel Oberle, and Steffen Staab. What is an ontology? In Handbook on ontologies, pages 1-17. Springer, 2009.

[2] Thomas R. Gruber. A translation approach to portable ontology specifications. Knowledge Acquisition, 5:199-220, 1993.

[3] Willem Nico Borst. Construction of engineering ontologies for knowledge sharing and reuse. PhD thesis, University of Twente, 1997.

[4] Rudi Studer, V Richard Benjamins, and Dieter Fensel. Knowledge engineering: principles and methods. Data \& knowledge engineering, 25(1-2):161-197, 1998.

[5] Matthew Horridge. A Practical Guide To Building OWL Ontologies Using Prot' eg' e 4 and CO-ODE Tools Edition 1.3. Technical report, Stanford, 2011.

[6] Thabet Slimani. Ontology development: A comparing study on tools, languages and formalisms. Indian Journal of Science and Technology, 8(24):1-12, 2015.

[7] Grigoris Antoniou and Frank Van Harmelen. Web ontology language: Owl. In Handbook on ontologies, pages 67-92. Springer, 2004.

[8] Juliao Braga, Joao Nuno Silva, Patricia Endo, and Nizam Omar. Structure for knowledge acquisition, use, learning and collaboration inter agents over internet infrastructure domains. In Kohei Arai, Rahul Bhatia, and Supriya Kapoor, editors, Intelligent Computing, volume 1, pages 527-547. Springer International Publishing, 2019. doi: 10.1007/978-3-030-22871-2, ISBN: 978-3-030-22871-2.

[9] Juliao Braga. Ambiente para Aquisição de Conhecimento por Agentes em Domínios Restritos na Infraestrutura da Internet. $\mathrm{PhD}$ thesis, Instituto Superior Técnico \& Universidade Presbiteriana Mackenzie, 2019. DOI: 10.31237/osf.io/nzmtf, Availaible in https://thesiscommons . org/nzmtf/.

[10] Juliao Braga. Environment for Knowledge Acquisition by Agents in Internet InfrastructureRestricted Domains. $\mathrm{PhD}$ thesis, Instituto Superior Tecnico \& Universidade Presbiteriana Mackenzie, 2019. DOI: 10.31237/osf.io/83ztf. English version translated by author. Available in https://thesiscommons.org/83ztf/.

[11] Mark A. Musen. The protégé project: a look back and a look forward. AI Matters, 1(4):4-12, 2015.

[12] Pascal Hitzler, Markus Krötzsch, Bijan Parsia, Peter F. Patel-Schneider, and Sebastian Rudolph. OWL 2 Web Ontology Language Primer. W3C Recommendation, 27(1):1-123, 2009.

[13] Diana Kalibatiene and Olegas Vasilecas. Survey on ontology languages. In International Conference on Business Informatics Research, pages 124-141. Springer, 2011.

[14] Christine Golbreich, Olivier Dameron, Bernard Gibaud, and Anita Burgun. Web ontology language requirements wrt expressiveness of taxonomy and axioms in medicine. In International Semantic Web Conference, pages 180-194. Springer, 2003.

[15] Tania Tudorache, Csongor Nyulas, Natalya F Noy, and Mark A Musen. Webprotégé: A collaborative ontology editor and knowledge acquisition tool for the web. Semantic web, 4(1):89-99, 2013.

[16] Adam Pease. Ontology. Articulate Software Press, 2011. ISBN: 1889455105. 
[17] Hoifung Poon and Pedro Domingos. Unsupervised ontology induction from text. In Proceedings of the 48th annual meeting of the Association for Computational Linguistics, pages 296-305, 2010.

[18] Hong Fu, Zheru Chi, Dagan Feng, and Jiatao Song. Machine learning techniques for ontology-based leaf classification. In ICARCV 2004 8th Control, Automation, Robotics and Vision Conference, 2004., volume 1, pages 681-686. IEEE, 2004.

[19] Feiliang Ren. A demo for constructing domain ontology from academic papers. In Proceedings of COLING 2012: Demonstration Papers, pages 369-376, 2012.

[20] Monica Shekhar and Saravanaguru RA. K. Semantic web search based on ontology modeling using protege reasoner, 2013.

[21] Philipp Cimiano and Johanna Völker. A framework for ontology learning and data-driven change discovery. In Proceedings of the 10th International Conference on Applications of Natural Language to Information Systems (NLDB), pages 227-238. Springer, 2005.

[22] Paul Buitelaar, Daniel Olejnik, and Michael Sintek. A protégé plug-in for ontology extraction from text based on linguistic analysis. In European Semantic Web Symposium, pages 31-44. Springer, 2004.

[23] He Tan and Patrick Lambrix. Selecting an ontology for biomedical text mining. In Proceedings of the BioNLP 2009 Workshop, pages 55-62, 2009.

[24] Dominik Sacha, Matthias Kraus, Daniel A Keim, and Min Chen. Vis4ml: An ontology for visual analytics assisted machine learning. IEEE transactions on visualization and computer graphics, 25(1):385-395, 2018.

[25] AnHai Doan, Jayant Madhavan, Pedro Domingos, and Alon Halevy. Ontology matching: A machine learning approach. In Handbook on ontologies, pages 385-403. Springer, 2004.

[26] Ryutaro Ichise. Machine learning approach for ontology mapping using multiple concept similarity measures. In Seventh IEEE/ACIS International Conference on Computer and Information Science (icis 2008), pages 340-346. IEEE, 2008.

[27] Paul Buitelaar, Philipp Cimiano, and Bernardo Magnini. Ontology learning from text: An overview. Ontology learning from text: Methods, evaluation and applications, 123:3-12, 2005.

[28] Muhammad Nabeel Asim, Muhammad Wasim, Muhammad Usman Ghani Khan, Waqar Mahmood, and Hafiza Mahnoor Abbasi. A survey of ontology learning techniques and applications. Database, 2018, 2018.

[29] Agnieszka Konys. Knowledge systematization for ontology learning methods. Procedia computer science, 126:2194-2207, 2018.

[30] Vaishak Belle and Ioannis Papantonis. Principles and practice of explainable machine learning. arXiv preprint arXiv:2009.11698, 2020.

[31] Tom Mitchell, William Cohen, Estevam Hruschka, Partha Talukdar, Bo Yang, Justin Betteridge, Andrew Carlson, B Dalvi, Matt Gardner, Bryan Kisiel, et al. Never-ending learning. Communications of the ACM, 61(5):103-115, 2018.

[32] Andrew Carlson, Justin Betteridge, Bryan Kisiel, Burr Settles, Estevam R Hruschka, and Tom M Mitchell. Toward an architecture for never-ending language learning. In Twenty-Fourth AAAI Conference on Artificial Intelligence, page 8, Atlanta, US, 2010. AAAI Publications.

[33] Daniel L Silver, Qiang Yang, and Lianghao Li. Lifelong machine learning systems: Beyond learning algorithms. In 2013 AAAI spring symposium series, 2013.

[34] Glauco Arbix. A trAnspArênciA no centro dA construção de umA iA éticA. Novos estudos CEBRAP, 39(2):395413, 2020.

[35] Mehrnoush Shamsfard and Ahmad Abdollahzadeh Barforoush. Learning ontologies from natural language texts. International journal of human-computer studies, 60(1):17-63, 2004.

[36] Giancarlo Guizzardi, Gerd Wagner, João Paulo Andrade Almeida, and Renata SS Guizzardi. Towards ontological foundations for conceptual modeling: The unified foundational ontology (ufo) story. Applied ontology, 10(34):259-271, 2015.

[37] Janez Brank, Marko Grobelnik, and Dunja Mladenic. A survey of ontology evaluation techniques. In Proceedings of the conference on data mining and data warehouses (SiKDD 2005), pages 166-170, Ljubljana, Slovenia, 2005. SiKDD.

[38] Guohui Xiao, Davide Lanti, Roman Kontchakov, Sarah Komla-Ebri, Elem Güzel-Kalayc1, Linfang Ding, Julien Corman, Benjamin Cogrel, Diego Calvanese, and Elena Botoeva. The virtual knowledge graph system ontop. In International Semantic Web Conference, pages 259-277. Springer, 2020. 
[39] Guohui Xiao, Linfang Ding, Benjamin Cogrel, and Diego Calvanese. Virtual knowledge graphs: An overview of systems and use cases. Data Intelligence, 1(3):201-223, 2019.

[40] Joaquim L. R. Dias Itana Stiubiener Juliao Braga, Francisco Regateiro. A machine learning domain ontology for populate knowledge base for support intelligent agents working in autonomous systems domains of the internet infrastructure. pages 1-12, 2021.

[41] Juliao Braga. Machine learning in the internet infrastructure project repository (mlii), Oct 2020. 